\title{
Memory in fungal pathogens promotes immune evasion, colonisation and infection
}

\author{
Alistair J.P. Brown*, Neil A.R. Gow, Adilia Warris and Gordon D. Brown \\ Medical Research Council Centre for Medical Mycology at the University of Aberdeen, \\ Institute of Medical Sciences, Foresterhill, Aberdeen, AB25 2ZD, United Kingdom. \\ * Correspondence: \\ Alistair J P Brown \\ E: al.brown@abdn.ac.uk \\ T: +44 (0)1224 437482
}

Key words: Fungal pathogenicity; immune evasion; fungal infection; fungal adaptation; adaptive prediction; fungal memory

\begin{abstract}
By analogy with Pavlov's dogs, certain pathogens have evolved anticipatory behaviours that exploit specific signals in the human host to prepare themselves against imminent host challenges. This adaptive prediction, a type of history-dependent microbial behaviour, represents a primitive form of microbial memory. For fungal pathogens, adaptive prediction helps them circumvent nutritional immunity, protects them against phagocytic killing, and activates immune evasion strategies. We describe how these anticipatory responses, and the contrasting lifestyles and evolutionary trajectories of fungal pathogens, have influenced the evolution of such adaptive behaviours, and how these behaviours affect host colonisation and infection.
\end{abstract}




\section{Microbial memory}

Microorganisms often inhabit dynamic niches where they are confronted by continuously fluctuating environmental challenges that are often perceived as stresses. Consequently, to survive, microbes have evolved adaptation mechanisms that mitigate against these challenges. Certain niches impose stresses that recur in a reasonably predictable manner in which one input is often followed by a second input of a certain type. This presents the resident microbes with an opportunity to develop anticipatory behaviours that have the potential to enhance their fitness in this niche. This is somewhat analogous to Pavlov's dogs, who were entrained to salivate upon hearing a bell in anticipation of being fed [1]. The rationale of adaptive prediction is that a microbe is likely to have a selective advantage over its competitors if it has evolved to activate a response to, and hence become protected against, a second impending input, when it is exposed to the initial (first) input [2] (Figure 1).

The extent of this selective advantage will depend upon the cost-benefits of the anticipatory gene expression mechanism. In other words, the energetic cost of the anticipatory response is weighed against the degree of protection it offers [3]. Theoretically, if the regulatory machinery comes at no cost to the organism, an optimal level of constitutive expression could provide higher fitness levels than responsive gene expression [4]. However, the significant cost of this machinery is illustrated by the down-regulation of the transcriptional and translational apparatus during times of stress [5]. Furthermore, elevating the basal levels of expression for certain stress factors can have a negative impact on other virulence traits [6]. Therefore, the expression of stress functions in the absence of stress does incur a fitness cost $[6,7]$. Nevertheless, clear examples of microbial stress priming have been reported, as summarised in this review and elsewhere [8], indicating that the fitness advantage conferred by some anticipatory responses outweighs their fitness cost.

The likelihood of developing an anticipatory response will also depend on the frequency and predictability of the relevant environmental challenges relative to the transient protection that the adaptive response confers (Figure 1). This implies that anticipatory behaviours probably become most entrained in organisms that experience specific environmental perturbations or rhythms or oscillations frequently and predictable within the niches they occupy.

Survival in dynamic environments can be enhanced via alternative mechanisms, such as noisy gene expression, switch-like behaviours or robust core stress responses. Nevertheless, examples of adaptive prediction have been reported in gut bacteria to take advantage of predictable changes in carbon source in the gastrointestinal tract, and in yeast to deal with sequential thermal, ethanol and oxidative stresses during wine fermentations [2]. 
The transcriptional programmes associated with circadian rhythms also provide excellent examples of anticipatory behaviours, tuning fungal metabolism and virulence traits to light dark cycles [9-13]. Historical behaviours can be evolved in vitro through the growth of Saccharomyces cerevisiae or bacteria under conditions that impose repetitive environmental changes $[2,3,14-16]$. It has been suggested that anticipatory behaviours such as these represent a primitive form of memory $[15,17,18]$.

Recent observations indicate that anticipatory behaviours are highly relevant to fungal pathogens and their interactions with the human host. The significant impact of fungal pathogens upon human health is generally underappreciated [19]. The main killers, Aspergillus fumigatus, Candida albicans, Cryptococcus neoformans and Pneumocystis jirovecii, have been estimated to kill over one million people each year, and dimorphic fungi, Histoplasma capsulatum, Coccidioides immitis, Blastomyces dermatitidis, Paracoccidioides brasiliensis, Sporothrix schenckii and Penicillium marneffei annually cause tens of thousands of deaths in North and South America and Asia [19]. The immune status of the individual strongly influences the incidence and/or severity of many infections caused by these fungi $[20,21]$. It is becoming clear that certain pathogenic fungi, and C. albicans in particular, have probably evolved anticipatory behaviours that address challenges posed by our immune defences. We discuss these anticipatory behaviours, the mechanisms by which they counteract immunity, and the probable basis for anticipatory behaviours in fungal pathogens.

\section{Evolutionary trajectories, lifestyles and anticipatory behaviours}

How likely is a fungal pathogen to have developed anticipatory behaviours that promote human colonisation and disease? This will depend on the lifestyle and evolutionary trajectory of the fungus, as well as the predictability of the inputs in host niches, and the cost-benefits to the fungus of the anticipatory behaviour.

Fungal pathogenicity has evolved independently in diverse branches of the fungal kingdom [22]. This implies that, with the exception of ancient environmental inputs such as the light-dark cycles underlying circadian rhythms [9-11], evolutionarily distant fungal pathogens have probably evolved different solutions to the common challenges they encounter in the human host, which include the ability to overcome or evade the immune system. It has been reported that adaptive prediction can emerge in relatively short evolutionary timescales in vitro (50-150 generations) [23]. Such behaviours are likely to take longer to emerge in natural niches because these environments are less prescriptive (in that the frequency and doses of natural stresses are variable), and more complex (fungal cells are exposed to multifarious inputs in their natural niches, in addition to the inputs of interest) than most in vitro microevolution experiments. Nevertheless, depending on the degree to 
which they confer an evolutionary advantage, anticipatory behaviours may have emerged relatively recently in evolutionary time. Taken together, this suggests that different anticipatory behaviours may have emerged in different pathogenic fungal species to address common challenges they face in the human host.

In evolutionary terms, $P$. jirovecii is highly tuned to its human host. This fungus has become so dependent on its intracellular niche that it has shed key metabolic pathways and cannot be cultured in vitro [24]. Despite the homeostatically buffered niche that it occupies, $P$. jirovecii could conceivably have evolved anticipatory behaviours that predict metabolic or stress responses in the host cell, for example. However, if such behaviours do exist in this fungus, they are likely to remain obscure for some time given the technical difficulties inherent in dissecting them in an organism that is currently non-culturable.

At the other extreme, some fungal pathogens are saprophytic, primarily occupying environmental niches. For example, $A$. fumigatus, $C$. neoformans, $H$. capsulatum, $C$. immitis and $B$. dermatitidis are abundant in the environment, and inhaled by individuals, causing lifethreatening infections in immunocompromised patients. While much attention is paid to their virulence factors, the evolutionary pressures that have shaped these fungi have been imposed in their environmental niches, not in humans. For example, the "grass eater", A. fumigatus is often viewed as an accidental pathogen that happens to possess properties that permit colonisation and infection in humans [25]. However, some fungal pathogens, Penicillium marneffei as well as Cryptococcus and Candida species for example, can infect animals, thereby providing potential reservoirs for human infection [26-28]. Clearly anticipatory behaviours of relevance to human infection may have evolved in these alternative hosts. Anticipatory behaviours that promote colonisation in humans may also have evolved in other environmental niches, for example through interactions with soil amoebae [29].

C. albicans is an interesting fungal pathogen, particularly from the perspective of the potential to evolve anticipatory behaviours within humans. Firstly, although there are reports of potential environmental reservoirs for this fungus [30,31], C. albicans is generally viewed as a commensal of humans, and is carried by many individuals in their orogastrointestinal and urogenital tracts [32,33], suggesting that it may have evolved in concert with its human host over recent evolutionary timescales. Secondly, the environmental responses of this fungal pathogen are relatively well characterised [34,35], raising the possibility that evidence of anticipatory behaviours might already exist for $C$. albicans.

\section{Anticipating nutritional immunity}


In an attempt to limit the proliferation of invading microbes, the mammalian host sequesters essential micronutrients, such as iron and zinc, via a process termed "nutritional immunity" [36-38]. A fungal pathogen must overcome the resultant micronutrient depletion by activating iron and zinc scavenging mechanisms if it is to proliferate and colonise these hosts [39-42]. Interestingly, in C. albicans, certain micronutrient scavenging mechanisms are linked to yeast-hyphal morphogenesis.

Morphological transitions play a key role in fungal virulence [43-46], and in C. albicans the yeast-to-hyphal transition is strongly associated with host invasion $[47,48]$. The formation of $C$. albicans hyphae can be stimulated by a variety of different experimental conditions, including exposure to bacterial peptidoglycan [49], but genome-wide transcriptional profiling has revealed a core set of only eight genes that are up-regulated during hyphal development under different hypha-inducing conditions [50]. Yet the formation of C. albicans hyphae is not dependent on hypha-specific genes [51].

These eight core hypha-specific genes in C. albicans include ALS3 and ECE1 [50]. Als3 promotes the assimilation of iron by scavenging ferritin, an iron storage protein, from the milieu [52]. ECE1 encodes candidalysin [53], a cytolytic peptide toxin that generates pores in host membranes and hence presumably makes ferritin more accessible to the invading fungus. Therefore, the induction of two genes involved in the acquisition of an essential micronutrient is hardwired to hyphal development (Figure 2). Yet these genes are not essential for hypha formation. Furthermore, the PRA1 and ZRT1 genes, which promote scavenging of another essential micronutrient, zinc [42], are up-regulated during $\mathrm{pH}$-induced hyphal development. Therefore, C. albicans induces iron and zinc scavenging mechanisms, just as it initiates hyphal growth and tissue invasion. Tissue invasion coincides with the imposition of nutritional immunity by the host as it attempts to limit fungal colonisation by limiting micronutrient availability $[38,54]$. Therefore, this appears to represent an excellent example of adaptive prediction, where the pathogen is essentially anticipating micronutrient deprivation before it invades micronutrient limiting domains [55,56] (Figure 2).

Iron starvation is a signal that triggers the activation of key virulence factors in C. neoformans, such as capsule formation [57]. This is likely to represent an anticipatory response (see Anticipating phagocytic recognition, below). In A. fumigatus, iron assimilation is integrated with $\mathrm{pH}$, oxidative stress and central carbon metabolism [58] rather than virulence factors per se [59]. Therefore, $C$. albicans appears to anticipate that micronutrient starvation will follow tissue invasion, and $C$. neoformans exploits this micronutrient starvation to trigger other virulence factors, whereas the saprophyte $A$. fumigatus simply triggers micronutrient scavenging mechanisms upon micronutrient starvation. 


\section{Anticipating phagocytic recognition}

Recent evidence suggests that fungal pathogens have also developed anticipatory behaviours that promote immune evasion in the host. The first step in the development of an immunological response against fungal pathogens involves their recognition by the innate immune system. Innate immune cells exploit a range of Toll-like receptors (TLRs) and Ctype lectin receptors (CLRs) to detect specific pathogen-associated molecular patterns, many of which are located at the fungal cell surface $[60,61]$. These include the canonical CLR, Dectin-1, which recognizes $\beta$-glucan [62], the mannose receptor [63], and MelLec, which recognises melanin [64]. The recognition of these pathogen-associated molecular patterns (PAMPs) leads to the activation of innate immune defences including phagocytic clearance, and the development of adaptive immune responses [63,65]. $\beta$-Glucan recognition by Dectin-1, in particular, plays a major role in antifungal immunity $[66,67]$, amplifying the response to other PAMPs [68]. The recognition of chitin by the intracellular receptors, TLR9 and NOD2, is thought to play a role in dampening inflammation once the fungal threat has been dealt with [69].

Changes in PAMP exposure at the fungal cell surface affect immune recognition and thereby influence the ability of a fungal pathogen to colonise its host. The degree of exposure of $\beta$-glucan on the $C$. albicans cell surface changes during morphogenesis and during infection [70-72] and affects competitive fitness in the gastrointestinal tract [73]. The degree of $\beta$-glucan exposure initially declines during deep-seated infections of internal organs, but then increases following neutrophil attack, thereby enhancing immune recognition $[71,74]$. What triggers the initial decline in $\beta$-glucan exposure on $C$. albicans cells during infection? Physiological levels of the carboxylic acid, lactate, have been shown to trigger the masking of $\beta$-glucan, which leads to attenuated cytokine responses and neutrophil recruitment [75]. Furthermore the virulence of $C$. albicans in the murine model of systemic candidiasis is enhanced by growth in the presence of lactate [76]. This $\beta$-glucan masking can be triggered by either host-derived or microbiota-derived lactate. It is activated via a non-canonical signalling pathway that has recruited key factors from signal transduction pathways involved in cellular morphogenesis and cell wall remodelling, namely the receptor Gpr1 and the transcription factor Crz1 [75]. Therefore, C. albicans would appear to have developed an anticipatory response to lactate that promotes fungal protection against subsequent immune attack in certain host niches (Figure 3).

$\beta$-Glucan masking can be triggered by a second host input, hypoxia, which leads to attenuated phagocytic recognition and uptake of $C$. albicans cells $[77,78]$. Hypoxia is imposed at infection sites through fungal-dependent neutrophil recruitment, which then leads to $\beta$-glucan masking and immune evasion [78]. As was observed for lactate-induced 
masking, evolutionarily conserved signalling modules have been recruited to drive this hypoxia-induced $\beta$-glucan masking. The response to hypoxia is dependent upon mitochondrial signalling, which then activates the cyclic AMP-protein kinase A pathway, leading to cell wall remodelling and $\beta$-glucan masking [77]. This suggests that, depending upon the host niche, $C$. albicans has evolved to exploit either of two host signals - hypoxia or lactate - to promote immune evasion (Figure 3).

C. albicans does not appear to mask cell surface $\beta$-glucan at all human infection sites. For example, the low ambient $\mathrm{pH}$ in vaginal niches trigger cell wall remodelling, which leads to the unmasking of underlying $\beta$-glucan in the $C$. albicans cell wall. This in turn leads to the enhanced release of inflammatory cytokines by macrophages, and increased neutrophil recruitment [79]. Accordingly, anticipatory responses that promote PAMP masking and immune evasion would appear to be relevant to some host niches but not others.

Other fungal pathogens mask PAMPs at their cell surface [80-82]. For example, Histoplasma, Paracoccidioides and Blastomyces increase the amount of $\alpha$-glucan in their cell walls during the process of cellular morphogenesis that generates their pathogenic yeast forms. The increase in a-glucan correlates with elevated virulence [83,84], perhaps due to immune avoidance. In the case of $H$. capsulatum, the a-glucan outer layer is thought to mask the immuno-stimulatory $\beta$-glucan in the cell walls [85]. During the development of the pathogenic yeast form, $H$. capsulatum also synthesizes the $\beta$-glucanase Eng 1 which, together with $\alpha$-glucan synthesis, promotes $\beta$-glucan masking and immune evasion [86]. $A$. fumigatus and $C$. neoformans also mask PAMPs at their cell surface. The melanin in the cell wall of $A$. fumigatus spores is masked by the hydrophobin, RodA, thereby protecting spores from MelLec-mediated recognition, the binding of host fibronectin, and phagocytosis $[64,87,88]$. C. neoformans synthesizes an elaborate capsule that masks the mannan and $\beta$ glucan in its cell wall [89]. The capsule is a key virulence factor for this pathogen, but the capsule does not simply represent an immune evasion mechanism as the capsule itself is recognised by TLRs [90]. Nevertheless, the fact that capsule formation is stimulated by iron depletion, suggests that $C$. neoformans has evolved an anticipatory response whereby nutritional immunity in the host triggers PAMP masking in anticipation of impending phagocytic attack. The master regulator Cir1 appears to control this anticipatory response by integrating iron sensing with capsule formation [57]. These fungi all cause debilitating infections in humans, but they exist primarily in environmental niches. Therefore, these anticipatory responses are likely to have developed as a result of evolutionary pressures in these microenvironments, possibly in non-human animal hosts, rather than specifically in human niches. 


\section{Anticipating phagocytic attack}

The ability of fungal pathogens to evade immune detection (above) was described by Underhill as "stealth" [81]. He also reviewed fungal mechanisms that promote "control" (mechanisms that modulate inflammatory processes) or "attack" (mechanisms that actively kill or counteract host defences). These mechanisms include the ability to bind complement regulatory proteins on the fungal cell surface $[91,92]$ or to degrade complement proteins $[93,94]$, thereby inhibiting the complement cascade. They include the development of growth forms that are recalcitrant to phagocytosis, such as $C$. neoformans Titan cells [44,95], C. immitis spherules, A. fumigatus germination and C. albicans hyphae, switching phenotypes and Goliath cells [96-99]. Even after phagocytosis, fungal pathogens are able to evade killing by perturbing phagolysosomal maturation and fungal killing [100-102], by escaping from macrophages by non-lytic vomocytosis [103,104], or by destroying macrophages via pyroptosis and other killing mechanisms [105-108]. In addition, many fungal pathogens activate robust stress responses to detoxify the reactive chemical species used by phagocytes to mediate microbial killing [34,109-111].

Although this has not been addressed explicitly, it is conceivable that some of these fungal behaviours are linked to anticipatory behaviours that have evolved in environmental niches or in the human host. For example, it is attractive to speculate that the induction of cryptococcal Titan cells in response to bacterially-derived peptidoglycan [95] might represent an example of adaptive prediction whereby exposure to an environmental stimulus promotes a fungal response that will protect it against subsequent amoebic attack. Similarly, the transcriptional programmes of dimorphic fungi include genes which are induced during the thermal transitions that induce their pathogenic forms, but which are not required for their growth at elevated temperatures. Some of these genes might represent further examples of anticipatory behaviours. For example, the expression of Blastomyces BAD1, an adhesin that suppresses pro-inflammatory responses, is induced in response to temperature [112]. In Coccidioides, the expression of the Mep1 metalloproteinase helps to mask an immunodominant cell surface antigen (SOWgp) during the development of pathogenic endospores, thereby enhancing the pathogenicity of this fungus [113]. Meanwhile the thermal induction of Cbp1 expression in Histoplasma subsequently causes alveolar macrophages that phagocytose the fungus to activate pro-apoptotic programmes, an essential step in the pathogenesis of this fungus [114]. Therefore, dimorphic fungal pathogens, which have evolved primarily in non-human niches in the environment, would appear to have developed potent anticipatory responses that enhance their virulence in the human host. 
Nevertheless, the following story [115], which links metabolic adaptation to immune evasion, provides a defined example of an anticipatory behaviour in a fungal pathogen that is likely to have evolved in the mammalian host.

In addition to the robust oxidative stress responses displayed by many fungal pathogens which protect them against phagocytic attack [34], these pathogens tend to display a high degree of metabolic flexibility, which enhances their ability to colonise divergent niches in their host and/or the environment [35,59,116-119]. An anticipatory behaviour in C. albicans was revealed by an unexpected link between metabolic and stress regulation in this fungus. In contrast to the benign "model yeast" Saccharomyces cerevisiae, in which glucose represses stress resistance [120,121], glucose enhances oxidative stress resistance in C. albicans [115]. Glucose concentrations are essentially zero in the colon, but when $C$. albicans transits into the bloodstream they are exposed to about $0.06-0.1 \%$ glucose [122]. Exposure to these physiological concentrations of glucose, or indeed plasma itself, is sufficient to trigger oxidative stress resistance in C. albicans [115]. Furthermore, the degree of oxidative stress protection is sufficient to provide protection against neutrophil killing [115]. This phenomenon is consistent with an anticipatory scenario where exposure to blood appears to be an early warning system for $C$. albicans, which then activates oxidative stress resistance mechanisms, and thereby becomes better protected against subsequent phagocytic attack (Figure 4).

A recent report reinforces the view that $C$. albicans has developed anticipatory behaviours that prepare the fungus for impending environmental change within its mammalian host [123]. C. albicans forms biofilms efficiently on abiotic surfaces such as catheters, which can become reservoirs of "dispersal" cells that seed bloodstream infection $[124,125]$. Interestingly, these dispersal cells display a transcriptional programme that includes features that appear to reflect future habitats rather than the biofilm environment from which they came [123]. For example, dispersal cells induce zinc and iron scavenging functions, apparently predicting nutritional immunity. Also, unlike their biofilm mother cells in the same rich medium, the dispersal cells express gluconeogenic and glyoxylate cycle genes [123], suggesting that they are primed to colonise glucose-poor niches in the host or to face phagocytic attack, during which glycoxylate cycle genes such as ICL1 are induced $[116,126]$.

\section{Conclusions and future perspectives}

It is becoming clear that fungal pathogens have evolved anticipatory behaviours that enhance their ability to counteract certain challenges they face in their human hosts. In C. albicans, this primitive form of memory helps the fungus to overcome the micronutrient 
depletion imposed by the host through nutritional immunity, to evade immune recognition by masking a key PAMP on its cell surface, and to combat phagocytic killing via prior activation of oxidative stress resistance mechanisms. Anticipatory behaviours may also be displayed by other fungal pathogens, which involve alternative mechanisms of immune evasion (see Outstanding Questions). Such behaviours will become more apparent as more investigators examine temporal correlations between phenotypes, rather than studying these phenotypes in isolation, and as they consider the in vivo context in which these dynamic adaptive responses occur.

The emergence of an anticipatory behaviour is no doubt dependent on the probability with which the relevant environmental inputs occur in the niche of interest, and the costbenefits of the behaviour to the fungus, but how might the regulation of the relevant adaptive responses become linked? (See Outstanding Questions.) This might be relatively straightforward, given the relative ease with which transcriptional networks can diversify [127], and the ease with which new protein kinase target sites can evolve on a regulatory protein [128]. These types of mechanism could potentially bring a stress regulon under the control of a new signalling pathway, for example [129]. Indeed, precedents for this exist in model yeasts. The analysis of $S$. cerevisiae strains that were able to anticipate transitions between carbon sources in vitro revealed that the adaptive mutations that drove this anticipatory behaviour lie in key transcriptional regulators [130]. Also, the emergence of regulatory mutations was sufficient to link normally unrelated phenotypes following the exposure of S. cerevisiae to repetitive environmental cues in vitro [23]. This is worthy of investigation in pathogenic fungi, because such mechanisms could account for the speed with which anticipatory behaviours can emerge, at least in vitro [23].

It has been said that the ultimate aim of research into host-fungus interactions is to translate observations about these interactions into novel therapies for patients who are vulnerable to lethal fungal infections [63]. A better understanding of anticipatory responses in fungal pathogens could potentially provide opportunities in this regard. For example, blocking the mechanisms by which specific host signals trigger PAMP masking by the fungus would, in principle, render the pathogen more vulnerable to immune clearance. Therefore, the characterisation of anticipatory responses in fungal pathogens could potentially reveal new therapeutic strategies as well as informing us about the nature of the dynamical challenges faced by fungal pathogens in host niches. These challenges represent more than the quest for food. Nevertheless, the fungal anticipatory responses that address these challenges are reminiscent of Pavlov's use of a bell to entrain salivatory responses in his dogs [1]. 


\section{Acknowledgements}

We are grateful to our colleagues in the medical mycology and fungal immunology communities for many stimulating and enjoyable discussions. We also thank Wai-Lum Sung for his help with the figures. The authors are directors of the Medical Research Council Centre for Medical Mycology at the University of Aberdeen led by GDB (MR/N006364/1). They are also funded by a Strategic Award from the Wellcome Trust [www.wellcome.ac.uk] led by NARG (097377). AJPB and NARG are funded by a programme grant from the UK Medical Research Council [www.mrc.ac.uk] (MR/M026663/1). NARG and GDB are supported by Wellcome Trust Investigator Awards (099215, 102705). NARG has additional awards from the Wellcome Trust $(075470,080088,086827)$. The funders had no role in study design, data collection and analysis, decision to publish, or preparation of the manuscript.

\section{References}

1. Pavlov, IP (1927) Conditioned reflexes: an investigation of the physiological activity of the cerebral cortex. London: Oxford University Press.

2. Mitchell, A. et al. (2009) Adaptive prediction of environmental changes by microorganisms. Nature $\underline{460}, 220-224$.

3. Mitchell, A. \& Pilpel, Y. (2011) A mathematical model for adaptive prediction of environmental changes by microorganisms. PNAS USA $\underline{108}, 7271-7276$.

4. Geisel, N (2011) Constitutive versus responsive gene expression strategies for growth in changing environments. PLOS ONE $\underline{6}$, e27033.

5. Gasch, AP (2007) Comparative genomics of the environmental stress response in ascomycete fungi. Yeast $\underline{24}$, 961-976.

6. Pradhan, A. et al. (2017) Elevated catalase expression in a fungal pathogen is a double-edged sword of iron. PLoS Pathogens $\underline{13}$, e1006405.

7. Markiewicz-Potoczny, M \& Lydall, D (2016) Costs, benefits and redundant mechanisms of adaption to chronic low-dose stress in yeast. Cell cycle $\underline{15}, 2732$ 2741.

8. Andrade-Linares, DR et al. (2016) Microbial stress priming: a meta-analysis. Environmental Microbiology 18, 1277-1288.

9. Idnurm A and Heitman J (2005) Light controls growth and development via a conserved pathway in the fun Idnurm, A. \& Heitman, J. (2005) Light controls growth and development via a conserved pathway in the fungal kingdom. PLoS Biol. $\underline{3}$, e95.

10. Ruiz-Roldan, M.C. et al. (2008) Role of the white collar 1 photoreceptor in carotenogenesis, UV resistance, hydrophobicity, and virulence of Fusarium oxysporum. Eukaryotic Cell ㄱ, 1227-1230.

11. Hevia, M.A. et al. (2015) A circadian oscillator in the fungus Botrytis cinerea regulates virulence when infecting Arabidopsis thaliana. PNAS USA 112, 8744-8749. 
12. Sancar, C. et al. (2015) Dawn- and dusk-phased circadian transcription rhythms coordinate anabolic and catabolic functions in Neurospora. BMC Biology $\underline{13}, 17$.

13. Mellor, J. (2016) The molecular basis of metabolic cycles and their relationship to circadian rhythms. Nature Struct Molec Biol 23, 1035-1044.

14. Tagkopoulos, I. et al. (2008) Predictive behavior within microbial genetic networks. Science 320, 1313-1317.

15. Wolf, D.M. et al. (2008) Memory in microbes: quantifying history-dependent behavior in a bacterium. PLoS ONE $\underline{3}$, e1700.

16. Dhar, R. et al. (2013) Yeast Adapts to a changing stressful environment by evolving cross-protection and anticipatory gene regulation. Molec Biol Evol $\underline{30}$, 573-588.

17. Casadesus, J. \& D'Ari, R. (2002) Memory in bacteria and phage. BioEssays $\underline{24}$, 512518.

18. Hellingwerf, K.J. (2005) Bacterial observations: a rudimentary form of intelligence? Trends Microbiol 13, 152-158.

19. Brown, G.D. et al. (2012) Hidden killers: human fungal infections. Science Trans. Med. 4, 165 rv13.

20. Casadevall, A. \& Pirofski, L.A. (2003) The damage-response framework of microbial pathogenesis. Nature Rev Microbiol 1 , 17-24.

21. Brown, G.D. \& Netea, M.G. (2007) Immunology of Fungal Infections. Springer. ISBN 978-1-4020-5491-4.

22. Taylor, J.W. (2006) Evolution of Human-Pathogenic Fungi: Phylogenies and Species. In "Molecular Principles of Fungal Pathogenesis" (Heitman J et al.; eds.) ASM Press, Washington DC, pp113-132.

23. Lopez Garcia de Lomana, A. et al. (2017) Adaptive prediction emerges over short evolutionary time scales. Genome Biol. Evol. 9 , 1616-1623.

24. Hoving, J.C. \& Kolls, J.K. (2017) New advances in understanding the host immune response to Pneumocystis. Curr Opin Microbiol. $\underline{40}$, 65-71.

25. Tekaia, F. \& Latge, J.P. (2005) Aspergillus fumigatus: saprophyte or pathogen? Curr Opin Microbiol $\underline{8}, 385-392$.

26. Staib, F. et al. (1985) A Cryptococcus neoformans strain from the brain of a wildlife fox (Vulpes vulpes) suspected of rabies: mycological observations and comments. Zentralbl. Bakteriol. Mikrobiol. Hyg. A 260, 566-571.

27. Edelmann, A. et al. (2005) Genetic relationship between human and animal isolates of Candida albicans. J Clin Micro 43, 6164-6166.

28. Cao, C. et al. (2011) Common reservoirs for Penicillium marneffei infection in humans and rodents, China. Emerg Infect Dis. 17, 209-214.

29. Casadevall, A. (2012) Amoeba Provide insight into the origin of virulence in pathogenic fungi. Adv Exp Med Biol $\underline{710}$, 1-10.

30. Irga, P.J. et al. (2016) Correspondence between urban bird roosts and the presence of aerosolised fungal pathogens. Mycopathologia 181, 689-699.

31. Bensasson, D. et al. (2018) Diverse lineages of Candida albicans live on old oaks. bioRxiv http://dx.doi.org/10.1101/341032. 
32. Sobel, J.D. (2007) Vulvovaginal candidosis. Lancet $\underline{369}$, 1961-1971.

33. Huseyin, C.E. et al. (2017) Forgotten fungi - the gut mycobiome in human health and disease. FEMS Microbiol Rev. 41, 479-511.

34. Brown, A.J.P. et al. (2014a) Stress adaptation in a pathogenic fungus. J. Exp. Biol. 217, 144-155.

35. Brown, A.J.P. et al. (2014) Metabolism impacts Candida immunogenicity and pathogenicity at multiple levels. Trends Microbiol 22, 614-622.

36. Corbin, B.D. et al. (2008) Metal chelation and inhibition of bacterial growth in tissue abscesses. Science $\underline{319}$, 962-965.

37. Urban, C.F. et al. (2009) Neutrophil extracellular traps contain calprotectin, a cytosolic protein complex involved in host defense against Candida albicans. PLoS Pathog $\underline{5}$, e1000639.

38. Potrykus, J. et al. (2013) Fungal iron availability during deep seated candidiasis defined by a complex interplay of systemic and local events. PLoS Pathogens $\underline{9}$, e1003676.

39. Ramanan, N. \& Wang, Y. (2000) A high-affinity iron permease essential for Candida albicans virulence. Science 288, 1062-1064.

40. Schrettl, M. et al. (2004) Siderophore biosynthesis but not reductive iron assimilation is essential for Aspergillus fumigatus virulence. J. Exp. Med. 200, 1213-1219.

41. Jung, W.H. et al. (2009) Role of ferroxidases in iron uptake and virulence of Cryptococcus neoformans. Eukaryotic Cell $\underline{8}, 1511-1520$.

42. Citiulo, F. et al. (2012) Candida albicans scavenges host zinc via Pra1 during endothelial invasion. PLoS Pathog $\underline{8}$, e1002777.

43. Klein, B.S. \& Tebbets, B. (2007) Dimorphism and virulence in fungi. Curr Opin Microbiol 10, 314-319.

44. Crabtree, J.N. et al. (2012) Titan cell production enhances the virulence of Cryptococcus neoformans. Infect. Immun. 80, 3776-3785.

45. Wang, L. \& Lin, X. (2012) Morphogenesis in fungal pathogenicity: shape, size, and surface. PLoS Pathog $\underline{8}$, e1003027.

46. Malavia, D. et al. (2017) Zinc limitation induces a hyper-adherent goliath phenotype in Candida albicans. Front. Microbiol $\underline{8}, 2238$.

47. Gow, N.A.R. et al. (2003) Infection of chick chorioallantoic membrane (CAM) as a model for invasive hyphal growth and pathogenesis of Candida albicans. Med Mycol 41, 331- 338.

48. Mayer, F.L. et al. (2013) Candida albicans pathogenicity mechanisms. Virulence $\underline{4}$, 119-128.

49. $\mathrm{Xu}, \mathrm{XL}$ et al. (2008) Bacterial peptidoglycan triggers Candida albicans hyphal growth by directly activating the adenylyl cyclase Cyr1p. Cell Host Microbe $\underline{4}, 28-39$.

50. Martin, R. et al. (2013) A core filamentation response network in Candida albicans is restricted to eight genes. PLOS ONE $\underline{8}$, e58613.

51. Naseem, S. et al. (2015) Hyphal growth in Candida albicans does not require induction of hyphal-specific gene expression. Molec Biol Cell 26, 1174-1187. 
52. Almeida, R.S. et al. (2008) The hyphal-associated adhesin and invasin Als3 of Candida albicans mediates iron acquisition from host ferritin. PLoS Pathog 4: e1000217.

53. Moyes, D.L. et al. (2016) Candidalysin is a fungal peptide toxin critical for mucosal infection. Nature 532, 64-68.

54. Crawford, A.C. et al. (2018) Biphasic zinc compartmentalisation in a human fungal pathogen. PLoS Pathog 14, e1007013.

55. Brunke, S. \& Hube, B. (2014) Adaptive prediction as a strategy in microbial infections. PLoS Pathog 10, e1004356.

56. Crawford, A.C. \& Wilson, D. (2015) Essential metals at the host-pathogen interface: nutritional immunity and micronutrient assimilation by human fungal pathogens. FEMS Yeast Research, 15, fov071.

57. Jung, W.H. \& Kronstad, J.W. (2008) Iron and fungal pathogenesis: a case study with Cryptococcus neoformans. Cell. Microbiol. 10, 277-284.

58. Schrettl, M. \& Haas, H. (2011) Iron homeostasis-Achilles' heel of Aspergillus fumigatus? Current Opin Microbiol 14, 400-405.

59. Kwon-Chung, K.J. \& Sugui, J.A. (2013) Aspergillus fumigatus-what makes the species a ubiquitous human fungal pathogen? PLoS Pathog $\underline{9}$, e1003743.

60. Netea, M.G. et al. (2015) Immune defence against Candida fungal infections. Nature Rev Immunol 15, 630-642.

61. Erwig, L.P. \& Gow, N.A.R. (2016) Interactions of fungal pathogens with phagocytes. Nature Rev Microbiol 14, 163-176.

62. Brown, G.D. \& Gordon, S. (2001) A new receptor for $\beta$-glucans. Nature $\underline{413}$, 36-37.

63. Netea, M.G. et al. (2008) An integrated model of the recognition of Candida albicans by the innate immune system. Nat. Rev. Microbiol. 6, 67-78.

64. Stappers, M.H.T. et al. (2018) Recognition of DHN-melanin by a C-type lectin receptor is required for immunity to Aspergillus. Nature $\underline{555}, 382-386$.

65. Dambuza, I.M. et al. (2015) Fungal recognition and host defense mechanisms. Microbiol Spectrum $\underline{5}$, FUNK-0050-2016.

66. Ferwerda, B. et al. (2009) Human dectin-1 deficiency and mucocutaneous fungal infections. N Engl J Med 361, 1760-1767.

67. Werner, J.L. et al. (2009) Requisite role for the dectin-1 beta-glucan receptor in pulmonary defense against Aspergillus fumigatus. J Immunol 182, 4938-4946.

68. Dennehy, K.M. \& Brown, G.D. (2007) The role of the $\beta$-glucan receptor Dectin-1 in control of fungal infection. J Leukocyte Biol 82, 253-258.

69. Wagener, J. et al. (2014) Fungal chitin dampens inflammation through IL-10 induction mediated by NOD2 and TLR9 activation. PLoS Pathog 10, e1004050.

70. Gantner, B.N. et al. (2005) Dectin-1 mediates macrophage recognition of Candida albicans yeast but not filaments. EMBO J 24, 1277-1286.

71. Wheeler, R.T. et al. (2008) Dynamic, morphotype-specific Candida albicans $\beta$-glucan exposure during infection and drug treatment. PLoS Pathog 4, e1000227. 
72. Lowman, D.W. et al. (2014) Novel structural features in Candida albicans hyphal glucan provide a basis for differential innate immune recognition of hyphae versus yeast. J Biol Chem $\underline{289}$, 3432-3443.

73. Sem, X. et al. (2016) $\beta$-glucan exposure on the fungal cell wall tightly correlates with competitive fitness of Candida species in the mouse gastrointestinal tract. Front Cell Infect Microbiol $\underline{6}, 186$.

74. Hopke, A. et al. (2016) Neutrophil attack triggers extracellular trapdependent Candida cell wall remodeling and altered immune recognition. PLoS Pathog 12, e1005644.

75. Ballou, E.R. et al. (2016) Lactate signalling regulates fungal $\beta$-glucan masking and immune evasion. Nature Microbiology 2 16238.

76. Ene, I.V. et al. (2012) Host carbon sources modulate cell wall architecture, drug resistance and virulence in a fungal pathogen. Cellular Microbiology 14, 1319-1335.

77. Pradhan, A. et al. (2018) Hypoxia promoting immune evasion by triggering $\beta$-glucan masking on the Candida albicans cell surface via mitochondrial and cAMP-protein kinase A signalling. mBio mBio01318-18.

78. Lopes, JP et al. (2018) Oxygen-deprivation enhances Candida albicans survival by evading immune surveillance. mBio mBio01529-18.

79. Sherrington, S.L. et al. (2017) Adaptation of Candida albicans to environmental pH induces cell wall remodelling and enhances innate immune recognition. PLoS Pathog $\underline{13}$, e1006403.

80. Netea, M.G. et al. (2003) Aspergillus fumigatus evades immune recognition during germination through loss of toll-like receptor-4-mediated signal transduction. $J$ Infect Dis.188, 320-326.

81. Underhill, D.M. (2007) Escape mechanisms from the immune response. In "Immunology of Fungal Infections" (G.D. Brown \& M.G. Netea, eds.) Springer, pp429442.

82. Marcos, C.M. et al. (2016) Anti-immune strategies of pathogenic fungi. Front. Cell. Infect. Microbiol. $\underline{6}, 142$.

83. Brandhorst, T.T. et al. (2002) Using new genetic tools to study the pathogenesis of Blastomyces dermatitidis. Trends Microbiol. 10, 25-30.

84. Puccia, R. et al. (2011) The Paracoccidioides cell wall: past and present layers toward understanding interaction with the host. Frontiers Microbiol 2, 257.

85. Rappleye, C.A. et al. (2007) Histoplasma capsulatum $\alpha$-(1,3)-glucan blocks innate immune recognition by the $\beta$-glucan receptor. Proc Natl Acad Sci USA $104,1366-$ 1370.

86. Garfoot, A.L. et al. (2016) The Eng1 $\beta$-glucanase enhances Histoplasma virulence by reducing $\beta$-glucan exposure. mBio $\underline{7}$, e01388-15.

87. Jahn, B. et al. (1997) Isolation and characterization of a pigmentless conidium mutant of Aspergillus fumigatus with altered conidial surface and reduced virulence. Infect. Immun. $\underline{65}$, 5110-5117.

88. Pihet, M. et al. (2009) Melanin is an essential component for the integrity of the cell wall of Aspergillus fumigatus conidia. BMC Microbiol. $\underline{9}, 177$. 
89. Cross, C.E. \& Bancroft, G.J. (1995) Ingestion of acapsular Cryptococcus neoformans occurs via mannose and beta-glucan receptors, resulting in cytokine production and increased phagocytosis of the encapsulated form. Infect. Immun. $\underline{63}, 2604-2611$.

90. Yauch, L.E. et al. (2004) Involvement of CD14 toll-like receptors 2 and 4, and MyD88 in the host response to the fungal pathogen Cryptococcus neoformans in vivo. Infect. Immun. 72, 5373-5382.

91. Zipfel, P.F. \& Skerka, C. (2009) Complement regulators and inhibitory proteins. Nature Rev Immunol $\underline{9}, 729-740$.

92. Zipfel, P.F. et al. (2013) Human complement control and complement evasion by pathogenic microbes - tipping the balance. Molec Immunol. 56, 152-160.

93. Gropp, K. et al. (2009) The yeast Candida albicans evades human complement attack by secretion of aspartic proteases. Molec Immunol. 47, 465-475.

94. Rambach, G. et al. (2010) Secretion of a fungal protease represents a complement evasion mechanism in cerebral aspergillosis. Molec Immunol. 47, 1438-1449.

95. Dambuza, I.M. et al. (2018) The Cryptococcus neoformans Titan cell is an inducible and regulated morphotype underlying pathogenesis. PLoS Pathog 14, e1006978.

96. Netea, M.G. et al. (2013) Aspergillus fumigatus evades immune recognition during germination through loss of Toll-Like Receptor-4-mediated signal transduction. J Infect Dis $\underline{188}, 320-326$.

97. Sasse, C. et al. (2013) White-opaque switching of Candida albicans allows immune evasion in an environment-dependent fashion. Eukaryotic Cell 12, 50-58.

98. Mukaremera, L. et al. (2017) Candida albicans yeast, pseudohyphal, and hyphal morphogenesis differentially affects immune recognition. Front. Immunol. $\underline{8}, 629$.

99. Crawford, A.C. et al. (2018) Biphasic zinc compartmentalisation in a human fungal pathogen. PLoS Pathog 14, e1007013.

100. Seider, K. et al. (2010) Interaction of pathogenic yeasts with phagocytes: survival, persistence and escape. Curr. Opin.Microbiol. 13, 392-400.

101. Morton, C.O. et al. (2012) Direct interaction studies between Aspergillus fumigatus and human immune cells; what have we learned about pathogenicity and host immunity? Front.Microbiol. $\underline{3}, 413$.

102. Smith, L.M. et al. (2015) The fungal pathogen Cryptococcus neoformans manipulates macrophage phagosome maturation. Cell Microbiol 17, 702-713.

103. Johnston, S.A. \& May, R.C. (2012) Cryptococcus interactions with macrophages: evasion and manipulation of the phagosome by a fungal pathogen. Cell Microbiol $\underline{15}$, 403-411.

104. Bain, J.M. et al. (2012) Non-lytic expulsion/exocytosis of Candida albicans from macrophages. Fungal Genet Biol 49, 677-678.

105. Uwamahoro, N. et al. (2014) The pathogen Candida albicans hijacks pyroptosis for escape from macrophages. mBio $\underline{5}$, e00003-14.

106. Wellington, M. et al. (2014). Candida albicans triggers NLRP3-mediated pyroptosis in macrophages. Eukaryotic Cell 13, 329-340.

107. O'Meara, T.R. et al. (2015) Global analysis of fungal morphology exposes mechanisms of host cell escape. Nature Comm $\underline{6}, 6741$. 
108. Tucey, T.M. et al. (2018) Glucose homeostasis is important for immune cell viability during Candida challenge and host survival of systemic fungal infection. Cell Metabolism 27, 988-1006.

109. Missall, T.A. et al. (2004) Mechanisms of resistance to oxidative and nitrosative stress: implications for fungal survival in mammalian hosts. Eukaryotic Cell $\underline{3}, 835-846$.

110. Campos, E.G. et al. (2005) Oxidative stress response in Paracoccidioides brasiliensis. Genet. Mol. Res. 4, 409-429.

111. Brown, A.J.P. et al. (2009) Nitrosative and oxidative stress responses in fungal pathogenicity. Curr. Opin. Microbiol. 12, 384-391.

112. Rooney, PJ et al. (2001) Selective expression of the virulence factor BAD1 upon morphogenesis to the pathogenic yeast form of Blastomyces dermatitidis: evidence for transcriptional regulation by a conserved mechanism. Molec Microbiol 39, 875-889.

113. Hung, CY et al. (2005) A metalloproteinase of Coccidioides posadasii contributes to evasion of host detection. Infect Immun $\underline{73}$, 6689-6703.

114. Isaac, DT et al. (2015) Macrophage cell death and transcriptional response are actively triggered by the fungal virulence factor $\mathrm{Cbp} 1$ during $H$. capsulatum infection. Molec Microbiol 98, 910-929.

115. Rodaki, A. et al. (2009) Glucose promotes stress resistance in the fungal pathogen, Candida albicans. Molec. Biol. Cell, 20, 4845-4855.

116. Lorenz, M.C. \& Fink, G.R. (2001) The glyoxylate cycle is required for fungal virulence. Nature $412,83-86$.

117. Kronstad, J.W. et al. (2011) Expanding fungal pathogenesis: Cryptococcus breaks out of the opportunistic box. Nature Rev Microbiol $\underline{9}$, 193-203.

118. Vylkova, S. et al. (2011) The fungal pathogen Candida albicans autoinduces hyphal morphogenesis by raising extracellular pH. mBio 2(3):e00055-11.

119. Childers, D.S. et al. (2016) The rewiring of metabolic ubiquitination targets in a pathogenic yeast promotes metabolic flexibility, host colonization and virulence. PLoS Pathogens 12, e1005566.

120. Gorner, W. et al. (1998). Nuclear localisation of the $\mathrm{C} 2 \mathrm{H} 2$ zinc finger protein Msn2p is regulated by stress and protein kinase A activity. Genes Dev. 12, 586-597.

121. Garreau, H. et al. (2000). Hyperphosphorylation of Msn2 and Msn4 in response to heat shock and the diauxic shift is inhibited by CAMP in Saccharomyces cerevisiae. Microbiology 146, 2113-2120.

122. Barelle, C.J. et al. (2006) Niche-specific regulation of central metabolic pathways in a fungal pathogen. Cellular Microbiology, $\underline{8}, 961-971$.

123. Uppuluri, P. et al. (2018). Candida albicans dispersed cells are developmentally distinct from biofilm and planktonic cells. (submitted).

124. Kojic, E.M. \& Darouiche, R.O. (2004) Candida infections of medical devices. Clin. Microbiol. Rev. 17, 255-267.

125. Finkel, J.S. \& Mitchell, A.P. (2011) Genetic control of Candida albicans biofilm development. Nature Rev Microbiol. $\underline{9}$, 109-118.

126. Lorenz, M.C. et al. (2004) Transcriptional response of Candida albicans upon internalization by macrophages. Eukaryotic Cell $\underline{3}, 1076-1087$. 
127. Baker, C.R. et al. (2012) Protein modularity, cooperative binding, and hybrid regulatory states underlie transcriptional network diversification. Cell 151, 80-95.

128. Holt, J.J. et al. (2009) Global Analysis of Cdk1 Substrate Phosphorylation Sites Provides Insights into Evolution. Science 325, 1682-1686.

129. Bleuven C \& Landry CR. (2016) Molecular and cellular bases of adaptation to a changing environment in microorganisms. Proc. R. Soc. B 283, 20161458.

130. Segre, AV et al. (2006) High-resolution mutation mapping reveals parallel experimental evolution in yeast. PLoS Biol. $\underline{4}$, e256.

\section{Figure Legends}

\section{Figure 1: Anticipatory responses in dogs and fungi.}

[A] Pavlov's dogs learned to associate sound (a bell ringing) with the likely provision of food, and consequently, they developed an anticipatory behaviour (salivation) upon receiving the initial signal (the ringing bell) [1]. By analogy, brewing strains of yeast have "learned", in the context of wine fermentations, to anticipate that an oxidative stress often follows a rise in temperature. Consequently they have developed an anticipatory transcriptional response whereby oxidative stress genes, as well as heat shock genes, are induced in response to heat shock [2]. [B] This type of anticipatory behaviour, which has been termed "adaptive prediction", leads to stress cross protection, whereby exposure to the first input (blue: e.g. a mild heat shock in S. cerevisiae) protects against subsequent exposure to a different type of input (red: e.g. oxidative stress) [2,34]. Upper graphs represent the adaptive transcriptional responses, whereas the lower graphs represent the fitness of cells following each stress. Note: In the absence of adaptive prediction, the initial blue stress only activates the corresponding blue genes, and the second red stress induces the expression of red genes (upper graphs). In contrast, during adaptive prediction, the initial response to the blue stress includes transcriptional adaptation of both blue and red genes (red and blue stripes), thereby enhancing fitness following the imposition of the second red stress (lower graphs).

\section{Figure 2: Anticipatory responses protect fungal pathogens against nutritional}

immunity. [A] In C. albicans, morphogenetic signals trigger hyphal development. Hyphal growth promotes fungal invasion into host tissues where nutritional immunity is activated to limit iron availability to the fungus [38]. The core set of genes that are induced during hyphal development include genes involved in the scavenging of essential micronutrient, iron [50]. [B] These genes include ALS3 and ECE1, which encode Als3 and candidalysin. Neither is essential for hyphal development, but ALS3 promotes, and ECE1 probably enhances, the 
scavenging by $C$. albicans of iron in the form of ferritin (yellow star). Candidalysin forms a pore that would allow the escape of ferritin from host cells, and Als3 promotes the uptake of ferritin into the fungal hypha [52]. Therefore, upon induction of hyphal growth $C$. albicans anticipates iron limitation and induces ferritin scavenging mechanisms.

Figure 3: Pathogenic fungi evade immune recognition via anticipatory responses.

[A] C. albicans cells expose $\beta$-glucan at their cell surface during the growth of new cell wall. $\beta$-Glucan is a key PAMP that is recognised by host PRRs to trigger antifungal responses by the innate immune system $[60,62]$. However, in response to specific host signals, such as lactate or hypoxia, $C$. albicans cells activate $\beta$-glucan masking $[75,77]$. [B] This reduces the recognition of fungal cells by phagocytes and attenuates the cytokine responses of neutrophils $[75,77,78]$.

Figure 4: Anticipatory responses protect fungal pathogens against killing by innate immune cells. [A] In S. cerevisiae, glucose represses stress resistance. In contrast, in C. albicans, glucose exposure leads to the activation of oxidative stress genes. As a result, glucose exposure protects $C$. albicans cells against a subsequent acute oxidative stress [115]. [B] Glucose also protects C. albicans cells against neutrophil killing [115], suggesting that, following exposure to blood, the fungal cells detect the glucose (yellow boat form) and activate an oxidative stress response to become resistant to this stress (red cell). This anticipatory response protects them against subsequent attack and killing by circulatory neutrophils.

\section{Highlights}

o Recent evidence indicates that fungal pathogens have evolved anticipatory behaviours.

o These anticipatory behaviours exploit specific signals in the human host to prepare the fungus for imminent host challenges.

o These anticipatory behaviours represent a primitive form of fungal memory.

o These anticipatory behaviours can activate micronutrient acquisition mechanisms before a fungal pathogen is exposed to nutritional immunity. Masking of pathogenassociated molecular patterns at the fungal cell surface can attenuate subsequent 
immune recognition. Stress responses can be induced to protect the fungus against impending phagocytic killing mechanisms.

o Together, these anticipatory behaviours promote immune evasion, fungal colonisation and infection.

\section{Outstanding questions}

o How common are anticipatory behaviours in fungal pathogens?

o To what extent do these behaviours promote fungal colonisation and infection in their human host or, alternatively, fitness in an environmental niche?

o What are the molecular mechanisms by which specific adaptive responses have become linked to create an anticipatory response?

o Do these mechanisms involve the extension of transcriptional regulons, or the addition of new regulatory targets for protein kinases, for example?

o Does the pharmacological inhibition of these anticipatory behaviours present a therapeutic opportunity? 
Figure 1: Brown et al.

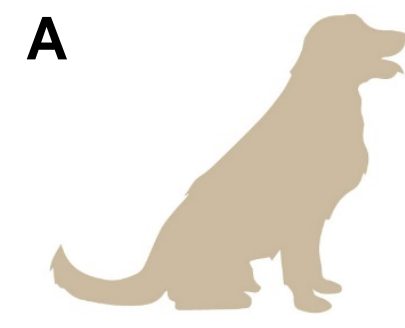

Pavlov's dogs

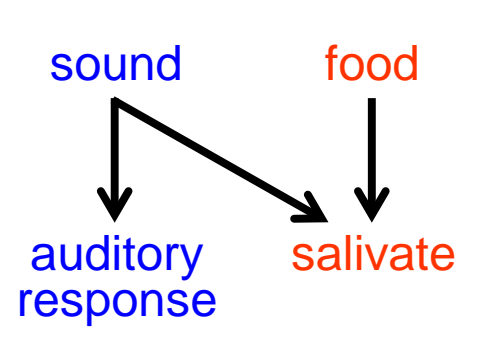

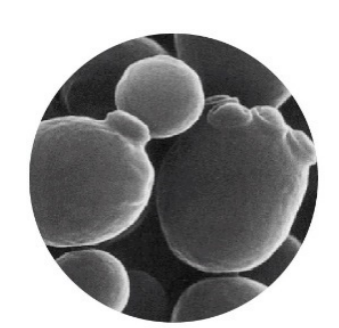

S. cerevisiae

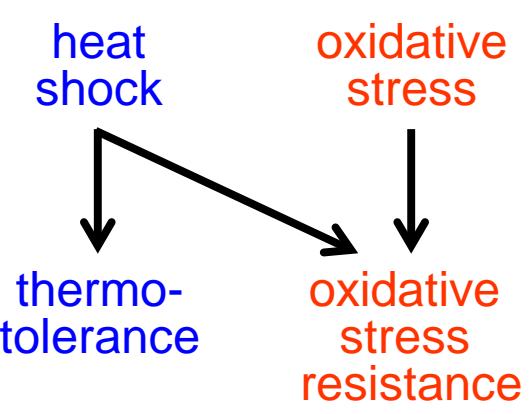

B no adaptive prediction
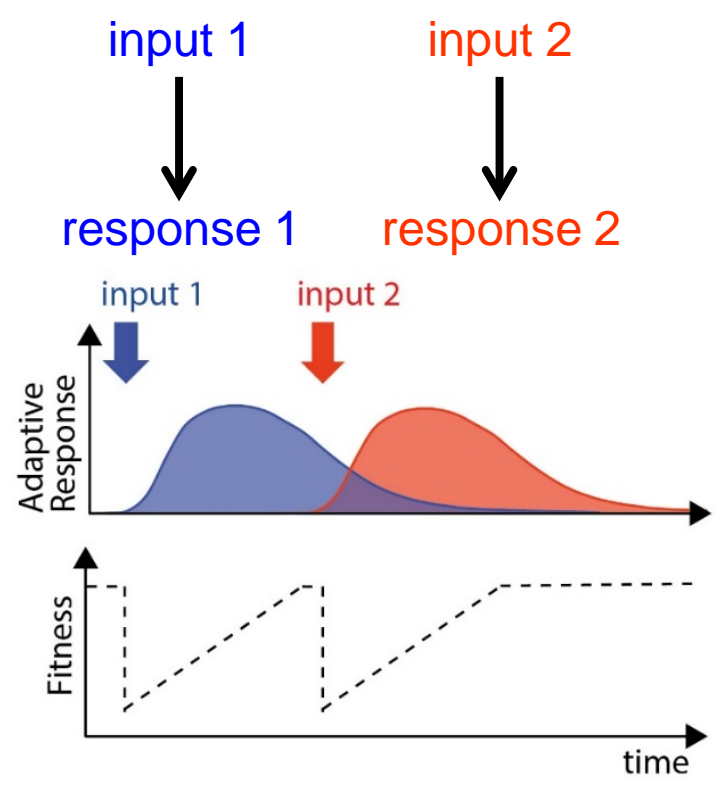

\section{adaptive prediction}

input 1 input 2

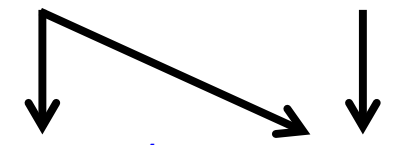

response 1 response 2
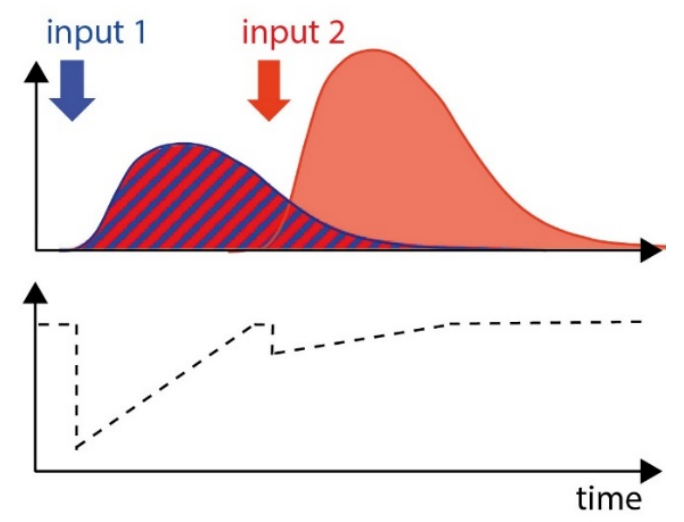
Figure 2: Brown et al.

A

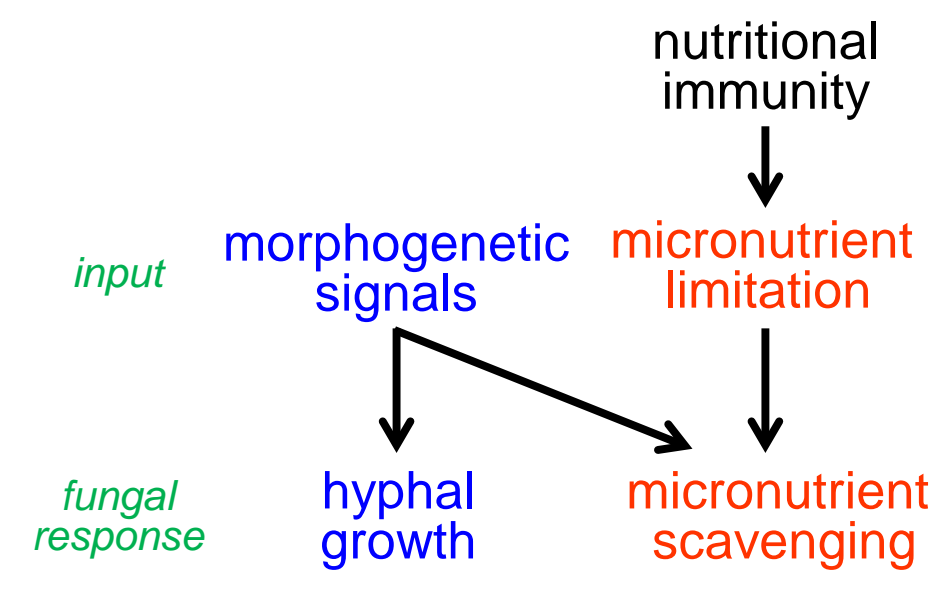

B

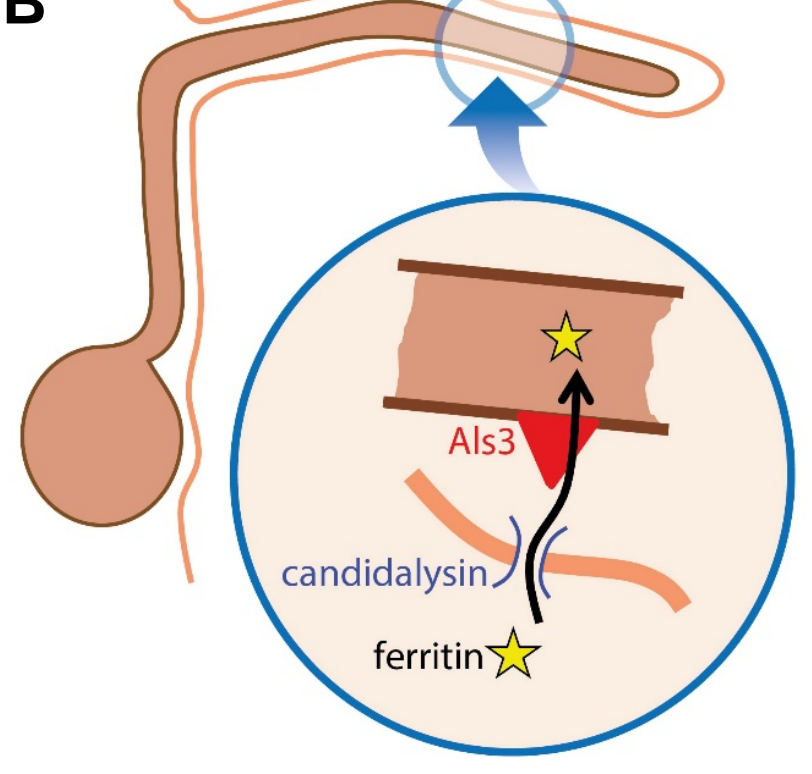


Figure 3: Brown et al.
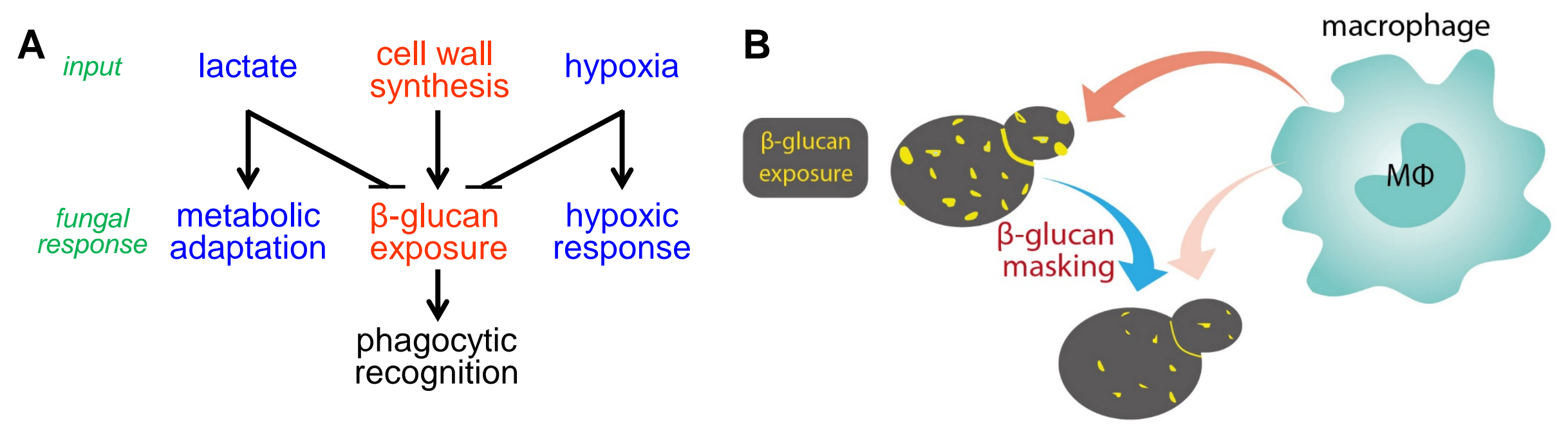
Figure 4: Brown et al.

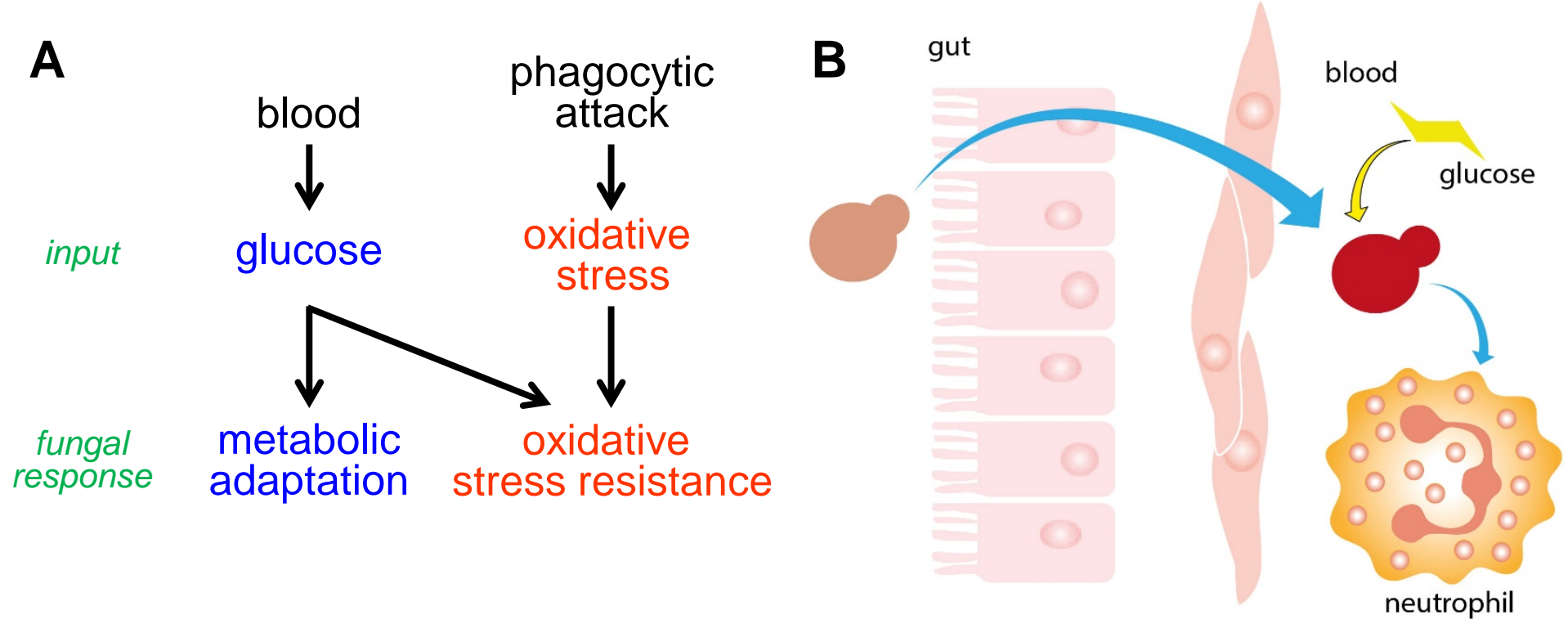

